\title{
Laser Joining of Continuous Glass Fiber Composite Pre-forms
}

\author{
Huade Tan, Y. Lawrence Yao \\ Department of Mechanical Engineering, Columbia University \\ New York, NY, USA
}

\begin{abstract}
A laser fusion joining method is investigated for the purpose of through thickness strengthening of fiber pre-forms used in the vacuum infusion fabrication of thick composite structures. Laser joining is achieved without filler materials to replace adhesives, pins or stitches used in conventional composite fabrication. A two step joining process is developed to fuse fibers within a single bundle and between multiple fiber bundles. Finite element analysis is used to investigate the joint strength with respect to joint morphology. Joint strength is found to be a function of the fiber contact angle and packing density at the joint interface. Tensile tests show that laser joined fiber bundles exhibit higher strength than comparable fastening methods. Lessons learned from the axial joining of fiber bundles are applied to joining in the radial and thickness directions of $3 \mathrm{~d}$ pre-form architectures. Flow induced joint morphology and densification effects observed in the axial direction indicate the need for a two step joining process in the thickness direction. Fiber compaction effects on joint strength in the axial direction motivate the need for high fiber packing fraction at joint interfaces in the thickness direction.
\end{abstract}

\section{INTRODUCTION}

Composite pre-preg fabrication involves placing densely packed resin infused tapes of reinforcement fibers in layers (laminates) and then curing to produce thin shell structures. Composite components manufactured from pre-preg processes exhibit high fiber packing fraction and high strength along the fiber directions, but offer little strength perpendicular to the fiber directions. Laminates of pre-preg construction contain no fiber reinforcements aligned in the thickness direction due to the layer by layer assembly process. Thus, pre-preg fabrication is undesirable for making composite parts requiring high through thickness strength and fracture toughness. One method for improving the through thickness strength of pre-preg constructed components is to insert metal pins through layers of pre-preg prior to curing [1,2]. Mechanical pinning increases through thickness strength, relying on matrix load transfer mechanisms between the fibers and pins. The insertion of metal pins into a stack of pre-preg lamina displaces the fibers in the lamina and introduces pockets of resin rich regions near the pins, which act as stress concentrations, resulting in reduced planar strength.

Composite fiber pre-form fabrication involves the assembly of fiber bundles into near form structures which are later infused and cured using vacuum resin infusion processes. Such methods have been shown to produce composites with desirable fiber packing and through thickness strength. Through thickness strength is achieved by distributing reinforcement fibers in the thickness direction using various stitching and weaving techniques [3-5]. The introduction of woven fibers or stitching materials in the thickness direction, however, also displaces fibers in the planar directions, similar to pinning. Woven pre-forms have been shown to yield significant improvements in out of plane material properties with some reduction of in plane properties [6,7].

Woven three dimensional fiber reinforcement pre-forms were adopted from textile processes as a replacement for laminate lay-up processes in applications requiring thick structures with improved through thickness strength $[5,6]$. In addition to improved mechanical properties, three dimensional woven pre-forms have been shown to improve fiber placement accuracy and reduce labor costs associated with ply lay-up vacuum infusion processes. A major process complication of $3 \mathrm{~d}$ woven pre-form fabrication, however, is the cost and complexity of the machinery required in the weaving process. The architecture or geometry of a $3 \mathrm{~d}$ woven pre-form is dictated by complex $3 \mathrm{~d}$ looms $[4,6]$. A $3 \mathrm{~d}$ woven pre-form composite of non constant thickness or irregular geometry thus requires expensive secondary machining steps after resin infusion and curing, adding to the cost of the process and impairing the strength of the material. A comparable process to $3 \mathrm{~d}$ weaving with a lower upfront cost and greater part flexibility is desirable.

Recently, the use of near infra red lasers to join thermoplastic fabrics $[8,9]$ has presented an alternative process to $3 \mathrm{~d}$ weaving, offering similar benefits at reduced complexity and cost. Textile applications that require high strength, high precision, seamless joints such as air bags, medical fabrics and protective garments are candidates for such a laser process. In these applications the added costs of laser joint processing are justified by high weld rates, localized joints, watertight sealing, and reduced labor costs. Laser joining of fibrous materials employs a direct fusion process unlike traditional 
mechanical or chemical bonding methods. Laser joining processes have been shown to offer better seam quality and strength compared to traditional stitching or weaving processes in fabrics. Similar to the adoption of $3 \mathrm{~d}$ weaving technologies from the textiles industry, the development of laser fiber joining presents a direct application to $3 \mathrm{~d}$ fiber preform fabrication.

Laser fusion joining presents possible advantages in strength over stitching, weaving or adhesion methods in its higher reinforcement density and direct fiber to fiber joining in the axial or radial directions. Laser processing allows joints to be restricted to highly localized regions, i.e. regions of existing stress concentrations. Laser fiber joining requires a lower upfront cost and allows for greater flexibility in part geometry and fiber architecture than $3 \mathrm{~d}$ weaving processes. Computer controlled manipulation and processing using laser irradiation to concurrently cut and join textiles reduces material waste and improves inventory efficiency. High weld rates may be achieved with current laser and optics technologies.

A physical challenge in the fusion joining of woven fibers, as observed in textile joining investigations [8-10], is the tendency of the melt pool to flow away from the desired joint region, forming voids in the processing zone. Both woven textiles and composite pre-forms are composed of many bundles of fibers, each of which is composed of hundreds of individual fibers. The relative density of a fiber bundle (fiber volume over total volume) is $50 \%$ to $90 \%$, depending on fiber packing. Due to fiber fusion and compaction processes during joining [11,12], the relative density of a fused joint is much greater than that of the initial fiber bundle. Thus the total volume of a fiber bundle required to form a joint is greater than the volume of the joint. During the fusion process, density gradient and capillarity effects between the melt and the fiber bundle drive bulk flows of the melt away from the high density regions of the joint toward the low density regions of the unprocessed fiber bundle. What results is the undesirable formation of voids, leading to poor load transfer and poor strength across the joint. In order to achieve fusion joining of $3 \mathrm{~d}$ fiber pre-forms, we must investigate the technical challenges inherent in the fiber fusion process.

One solution to both the relative density and flow problem has been to introduce a secondary filler material into the joint. Filler materials of desirable absorption properties at $1064 \mathrm{~nm}$ wavelength, developed for transparent lap joining of thermoplastic materials, have been used successfully in the laser lap joining of thermoplastic fabrics $[8,10]$. Such fillers are composed of proprietary polymers, adding cost and complexity to the joining process. No such filler materials have been developed for glass. A similar solution to overcoming the fiber spacing and flow problems is required to accomplish fiber fusion joining of glass reinforcements.

The objective of this study is to investigate the laser joining of glass fiber pre-form structures without using filler materials. A two step laser joining process for glass fiber reinforcement pre-forms is developed to overcome the relative density and flow induced void formation problem. This process compensates for the compaction and flow behavior of the fiber bundle during fusion and uses only the existing fiber material to achieve dense joints. Numerical solutions using a two phase immiscible Lattice Boltzmann Equation (LBE) model are used to reproduce the melt flow pattern and joint morphology during a fusion joining process. LBE modeling is used to characterize the effects of surface tension and viscosity and determine the relative time dependence of the melt flow behavior. Finite element analysis is performed to determine the effect of joint morphology on local stress concentration factors. An extension of the joining process into two dimensional plain weave fabric materials is performed to illustrate the application of fusion joining to the processing of $3 \mathrm{~d}$ pre-forms. Axial joint morphologies are investigated in this work for the purpose of experimental simplicity using a single bundle. Through thickness joined $3 \mathrm{~d}$ pre-forms may be constructed from the repeated joining of layers of woven fiber reinforcements in the radial direction. Radial fiber joints may be achieved in the same two step process outlined in this work. Lessons learned from the contact angle and packing density effects on joint strength observed from single bundle tensile tests may be applied to radial joints within a through thickness reinforced $3 \mathrm{~d}$ pre-form. It is indicated from single bundle tests that by substituting mechanical fasteners such as pins, stitches or weaves for fusion joining in the thickness direction, through thickness strength on the order of the glass fiber reinforcement may be achieved.

\section{EXPERIMENTAL SETUP}

Laser joining of e-glass fibers was achieved using a $2 \mathrm{~kW}$ $\mathrm{Nd}$ :YAG laser operating at a continuous range of $30 \mathrm{~W}$ to 120W. All glass samples consist of $99.98 \%$ E-glass plain weave fabric. Samples were prepared from six ounce per yard plain woven fabric with 18 bundles of fiber per inch and 300 fibers per bundle. The Gaussian laser spot was defocused to a diameter of $2 \mathrm{~mm}$ to allow for more uniform irradiation of a single fiber bundle. Motion control was accomplished using a six axis robotic manipulator with a flat, square grid sample holder to facilitate precise fiber placement.

Tensile testing of single bundle and seam welded plain weave samples were performed with a $2 \mathrm{kN}$ load cell at a rate of 0.5 millimeters per second. Single bundle fiber tension tests were performed using a grooved cylindrical grip system. Each sample was composed of two nine inch long fiber bundles joined in the center, forming an 18 inch long sample. Fiber bundle samples were wound around the grooves in each grip such that the joint was centered between the grips and loaded in tension. Plain weave tensile tests were conducted using flat grips. Gripping areas were cured in epoxy prior to testing to provide load transfer between the grips and the woven fibers.

Joint and fiber fracture surfaces were imaged using SEM post fracture to evaluate the failure mechanisms during tensile testing. Qualitative observations of the fracture surfaces were compared to the stress concentrations results obtained from a finite element model and observations during the test to determine sources of stress concentrations and the cause of joint failure.

E-glass is highly transmitting at the $1064 \mathrm{~nm}$ wavelength. It was observed that densely packed E-glass fibers are of 
sufficient opacity to initiate significant heat accumulation. Infrared absorption of highly transmitting materials due to multiple surface scattering has previously been observed in investigations of densely packed beds of glass spheres [13] and porous media [14]. Absorption of glass is also known to be highly dependent on temperature $[15,16]$. Glass simultaneously absorbs and emits a wide range of wavelengths near the phase transition temperature $[17,18]$.

Although other laser textile applications have evaluated the use of absorptive coatings for heat accumulation, it was determined from initial irradiation trials that surface coatings induce uneven absorption and poor joint density. Chemical reactions and oxidation occurs in fibers with surfacing agents owing to the localized heating area and the dramatic increase in laser absorption at elevated temperatures [15,18]. Temperature becomes uncontrollable once vaporization initiates. If the melt pool is allowed to exceed the vaporization temperature, ionization quickly follows, and the resulting plasma consumes the entire melt pool and any surrounding fiber material within a few spot radii. Uniform thermal absorption throughout the target spot is desired.

The dominant material transport process for glass fusion and densification processes is viscous flow and capillarity, as cited in glass sintering processes $[19,20]$. As the melt volume increases, gravitational force becomes significant compared to the capillary and viscous forces. A particular challenge to fusion joining of fibrous systems is the high surface area and low relative density of the initial material. The joining method devised in this work is derived from the need to accommodate for viscous flow effects inherent in the fiber system.

\section{NUMERICAL SIMULATION}

\section{Fluid Flow Simulation}

To simulate the flow behavior of the molten pool during fiber joining, a two phase immiscible Lattice Boltzmann Equation (LBE) model is implemented. Lattice Boltzmann methods are particularly useful in simulating low Reynolds number fluid flows through complex boundary conditions and multi-phase interactions [21]. An LBE multi-phase immiscible flow model is used to represent the glass and air phases with boundary lattices representing the solid glass phase. Phase evolution output from the LBE model post processing yield the time dependant flow of glass in both the beading and joining processes.

The main assumptions of the LBE model are: immiscible (no mixing between glass and air phases), incompressible (constant volume), uniform viscosity and surface energy, and negligible body forces. Immiscible fluid flow between molten glass and air assumes the vaporization and evaporation effects of glass may be considered negligible; which is reasonable in a fusion joining application, where the bulk temperature remains below the vaporization temperature. Fluid incompressibility is valid for the molten glass phase. Fluid properties such as viscosity and surface energy of glass are highly dependent upon glass temperature, which may not be uniform throughout the melt pool. This assumption, however, is inherent in this LBE formulation and is the main limiting factor of this model. Lastly, the negligible body force assumption is applicable when the length scale of the weld joint is on the order of the fiber diameter. As the size of weld pool exceeds approximately twice the bundle diameter, where the weight of the pool exceeds the viscous and surface tension forces of the fluid, the flow will begin to be affected by gravity. For cases where the melt diameter is less than this critical diameter, it is reasonable to neglect body forces.

The two dimensional immiscible LBE model used in this investigation was developed from the works of Gunstenssen, Rothman and Lishchuck et al [22-24]. The immiscible two phase LBE formulation solves the local collision equations

$$
-\boldsymbol{f} \cdot \boldsymbol{q}\left(r^{\prime}, b^{\prime}\right)=\min _{r^{\prime \prime}, \mathrm{b} "}-\boldsymbol{f} \cdot \boldsymbol{q}\left(r^{\prime \prime}, b^{\prime \prime}\right),
$$

where primes and double primes denote the lattice update iteration subject to mass and momentum conservations

$$
\begin{aligned}
& \sum_{i} r_{i}^{\prime \prime}=\sum_{i} r_{i}, \sum_{i} b_{i}^{\prime \prime}=\sum_{i} b_{i}, \\
& \sum_{i} c_{i}\left(r_{i}^{\prime \prime}+b_{i}^{\prime \prime}\right)=\sum_{i} c_{i}\left(r_{i}+b_{i}\right),
\end{aligned}
$$

on the lattice scale where $\boldsymbol{f}$ is the momentum density or direction weighted sum of the opposing phase velocities, $\boldsymbol{q}$ is the difference in net momentum between the two phases, $c$ is the lattice velocity, and $r$ represents the first phase while $b$ represents second phase. At the limit where the lattice size approaches zero relative to the solution space, the LBE formulation recovers the incompressible Navier Stokes equations [21]

$$
\begin{aligned}
& \rho \partial_{t} \boldsymbol{u}+\rho(\boldsymbol{u} \cdot \nabla) \boldsymbol{u}=-\nabla p+\mu \nabla^{2} \boldsymbol{u}, \\
& \nabla \cdot \boldsymbol{u}=0,
\end{aligned}
$$

where $\boldsymbol{u}$ is the fluid velocity field, $\rho$ is the fluid density and $\mu$ is the dynamic viscosity subject to equivalent normal fluid velocities along the phase interface

and surface tension

$$
\boldsymbol{u}_{1} \cdot \boldsymbol{n}=\boldsymbol{u}_{2} \cdot \boldsymbol{n}=\boldsymbol{u}_{\text {int }} \cdot \boldsymbol{n}
$$

$$
\boldsymbol{T}_{2} \cdot \boldsymbol{n}-\boldsymbol{T}_{1} \cdot \boldsymbol{n}=\left(\frac{\sigma}{R}\right) \boldsymbol{n},
$$

on the macro scale. A D2Q9 lattice is used in this Rothman Lishchuk model with two dimensions and nine velocity vectors [21]. Following Lishchuck's formulation, the immiscible LBE momentum density update is written as a single phase with an added pressure field

$$
\boldsymbol{f}_{i}\left(\boldsymbol{r}+\boldsymbol{c}_{i} \delta_{t}, t+\delta_{t}\right)=\boldsymbol{f}_{i}(\boldsymbol{r}, \boldsymbol{t})+\frac{1}{\tau}\left(\boldsymbol{f}_{i}^{(0)}-\boldsymbol{f}_{i}\right)+F_{i},
$$

where $F_{i}$ is the surface tension perturbation at the phase interface given by

$$
F_{i}=\frac{\alpha}{c_{s}^{2} R} t_{p} c_{i j} \partial_{j}\left(\frac{\rho^{N}}{2 \rho}\right)
$$

In this formulation, the local kinematic viscosity and surface tension parameters are defined by $v=\frac{2 \tau-1}{6} \delta_{t}$ and $\alpha$, where $\delta_{t}$ is the lattice time step, $t_{p}$ and $c_{s}^{2}$ are lattice dependent weights, $\tau$ is the lattice time constant, $\rho$ is the phase mass, $\rho^{N}(\boldsymbol{r}, t)=\rho_{r}(\boldsymbol{r}, t)-\rho_{b}(\boldsymbol{r}, t), \quad$ and $c_{i \mu}$ are velocity vectors defined by the D2Q9 lattice [23]. Lishchuck's formulation clearly defines the fluid viscosity and surface tension of each fluid phase. It has been shown to generate smoother velocity profiles at the phase interface than Gunstensen and Rothman's formulation.

\section{Mechanical Model}

A linear elastic, static finite element analysis $(\sigma=E: \epsilon)$ of a spherical body connected to a cylindrical bundle is used to simulate the stress state in a fiber joint (Figure 1). Finite 
element simulations of various idealized joint geometries under uni-axial tensile strain are performed to determine the stress concentration factor in the fiber bundle near the bead joint. The spherical bead joint is assumed to be composed of a solid isotropic linear elastic material with the modulus and strength of soda lime glass. The cylindrical fiber bundle is defined to be a linear elastic orthotropic material consistent of glass fiber reinforced composite aligned to the y axis (material coordinate reference 1 in Figure 1) defined by engineering constants $E_{i}, G_{12}, G_{13}, G_{23}$ and $v_{i j}$, where $\frac{v_{i j}}{E_{i}}=\frac{v_{j i}}{E_{j}}$. For computational simplicity, one eighth of the joint geometry is modeled. Symmetric boundary conditions are applied to the bisecting $(\mathrm{x}-\mathrm{y}, \mathrm{y}-\mathrm{z})$ planes.

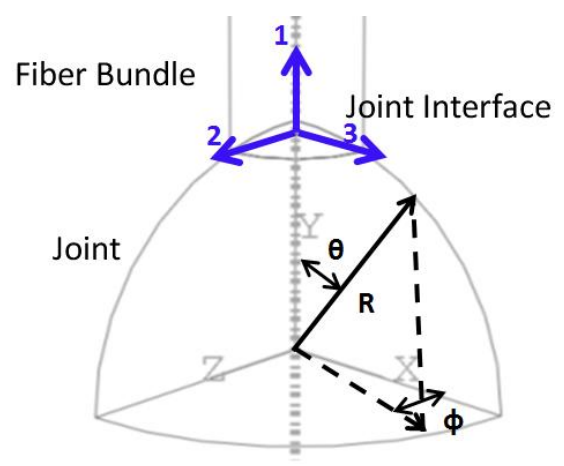

Figure 1: Finite element model outline and spherical coordinate axes. For computational simplicity, one eighth of the joint geometry is modeled with symmetric boundary conditions on each of the dividing planes.

The length of the entire model is set to 10 times the constant bundle diameter and the diameter of the sphere is varied to obtain different contact angles. The contact angle is defined as the maximum angle $\theta$ from the y axis at which the cylinder intersects the sphere. A zero contact angle thus corresponds to a sphere of infinite radius and a 90 degree contact angle corresponds to a sphere of the same radius as the bundle.

The nodes on the $\mathrm{x}-\mathrm{z}$ plane are fixed in the $\mathrm{y}$ direction only. A uniform finite displacement of $0.02 \%$ of the bundle diameter is applied to the nodes at the opposite end of the cylinder. The corresponding strain $(\epsilon=\Delta L / L)$ of $2 \mathrm{e}-5$ is applied on the entire assembly. The spatially resolved stress state at the interface of the cylindrical bundle and spherical bead is obtained from each finite element simulation for distinct contact angles. From these numerical results, the stress concentration factor $K$ is defined as

$$
K=\frac{\sigma_{\operatorname{Max}}}{\sigma_{0}}
$$

where $\sigma_{\text {Max }}$ is the maximum principle stress component at the joint perimeter and $\sigma_{0}$ is the uni axial stress state of a uniform fiber bundle at the same strain. Although neither the fiber bundle nor joint is homogenous or entirely dense, this continuum elastic model is used to determine the effect of the material isotropy mismatch during tensile testing on the stress concentration.

\section{RESULTS AND DISCUSSION}

Joining of glass fiber structures was achieved through a two step process depicted in Figure 1. The first step is to fuse fibers within a bundle into a dense bead by irradiating the free end(s) of the bundle and scanning along the fiber axis. These beads serve three purposes in the joining process. Fused beads provided the excess glass material to be consumed by the joint during later processing. This attribute is crucial in the formation of dense joints rather than voids in the process zone. Fused beads were found to be selectively absorbing of $1064 \mathrm{~nm}$ laser irradiation in later processes, allowing for greater control in the macro scale joining process. Lastly, by fusing the glass fibers in a single bundle, beads assist in the placement and alignment of the samples to be joined.

In the second step of the joining process, the beads are brought into contact and irradiated to form a solid joint. The total volume of the joint is determined by the initial volume of both beads. The morphology and compaction of the fiber bundles is determined by the mechanical constraints acting on the fiber bundles during joining. Joining of both single bundles and bundles in the plain weave configuration is performed. Single bundle joints consist of two initially separate bundles joined end to end using the process in Figure 2. Plain weave seam joint samples consist of two sheets of fabric joined along a common edge by the continuous repetition of single bundle joints between each opposing bundle pair.

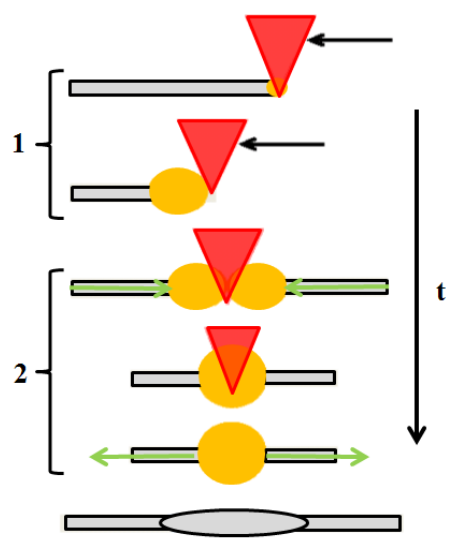

Figure 2: Laser fusion joining process schematic. Step 1: initiate fiber compaction and melt pool formation within a single bundle. Scan along bundle axis at a constant speed to accumulate melt volume. Step 2: Form joint between bundles by contacting initially formed beads. Apply tension to compress fibers in radial direction.

\section{Bead Formation}

The primary role of bead formation is to increase the relative density of the glass at the desired joint location. Upon laser irradiation, rapid heat accumulation occurs due to boundary and temperature dependant absorption and poor thermal conductivity of the glass fibers. Fiber joining is observed to initiate at the center of the focal area, where the laser intensity is highest, and propagates rapidly outward. Once the temperature of the fibers is sufficient to initiate flow, rapid fiber to fiber joint formation occurs between nearest 
neighbors within the fiber bundle. This process quickly collects all fibers within the laser spot to form a single pool of molten glass. The bead formation process initiates upon laser exposure of the fiber bundle. The absorption of laser energy is observed from the emission of glass in the visible range, from faint yellow and orange to white.

Joining of the glass fibers is observed as the reduction of bundle dimensions at the center of focus, resulting in rapid shortening of individual fibers and contraction in bundle diameter. Fibers quickly shorten and agglomerate to lower their surface energy [25]. The glass material at this point is highly emissive and fluid with microscopic flow patterns dominated by viscous joining effects. At this stage, the energy required to overcome viscous forces are provided both by the laser and the surface energy of the fiber compact $[11,19]$. The molten flow is driven toward both the center of the focus and the highest glass density. The rapid formation of a pool of molten glass is observed at the center of the focal point. Flow of molten glass takes place as nearby fibers are pulled into the melt pool. The melt pool collects into a spherical bead to minimize surface energy. The bead diameter is a function of the total volume of fiber consumed during this process.

As the diameter of the bead approaches the bundle diameter, the microscopic flow patterns at the fiber/melt interfaces become aligned in the same direction along the bundle axis. There exists a sharp density gradient along the melt interface between the melt and the fiber bundle. Similar to anisotropic sintering applications, bulk flow occurs in the direction of the greatest density gradient $[11,26]$. At this point the viscous flow behavior on the micro scale becomes observable on the macro scale as the bulk flow of the entire bead. Taking from Scherer's work on cell based sintering $[11,25]$, if an idealized unit cell of unidirectional fiber compacts is assumed, a bulk flow strain is obtained along the fiber axis.

After initial melt formation, the absorption of the pool is sufficient to allow the flow to remain molten near the laser spot. If the spot remains stationary, the melt pool will begin to flow away from the center of the spot temperature of the pool. There exists a point near the edge of the spot where the viscosity and capillary forces acting on the pool balance and the melt achieves a static equilibrium. If the beam or the sample is translated at a constant velocity along the bundle axis, the bulk flow will continue to propagate along the bundle in a quasi-static manner.

If the center of a bundle is irradiated such that both free ends are too far for surface tension effects to overcome frictional forces acting on the bundle, or if the bundle is constrained from translating on both ends, the melt pool will be pulled in both directions and be split in two. This process results in the formation of two bundles with opposing bead ends. This undesirable behavior, depicted in Figure 3, is the root cause of the void formation and connectivity problems observed in laser textile joining applications [8-10].

If irradiating sufficiently close to the free end of a single bundle, the molten bead forms at the planar focus and all excess fiber material between the bead and the free end is quickly consumed by the bead, resulting in a fiber with a single bead attached to the free end of the bundle. Upon scanning along the fiber axis, the bead volume may be controlled as shown in Figure 4. In this case the surface tension of the melt pool is sufficient to pull all nearby fibers into the melt pool. Stable bead formation was obtained with a scanning speed of $0.5 \mathrm{~mm} / \mathrm{s}$ at an average laser output of $110 \mathrm{~W}$. Bead diameters of twice the bundle diameter were achieved after a scanning distance of $3 \mathrm{~mm}$.

It is determined in this work that the joining of pre-form structures is primarily controlled by the relative fiber density and fiber orientation at the desired joint location. Flow of the melt pool is captured by the viscous flow of a fluid through a porous media defined by axially aligned fibers using the LBE method developed by Rothman and Lischuk et al $[22,23]$. By defining the capillarity driven viscous flow of the bead transport behavior as a boundary value problem with constant fluid properties, a multiphase immiscible LBE model can be shown to reproduce both bead formation and separation behaviors. The bead formation along a free end of the bundle obtained from LBE is depicted in Figure 4R. Bundle separation at the center of a fiber bundle is depicted in Figure 3R. The bead diameter time history of both processes is depicted in Figure 5. Although this simple LBE model ignores the effect of temperature variation on the fluid properties and does not consider the phase change between solid to liquid glass, the bulk behavior of the flow shows good agreement with experiments.

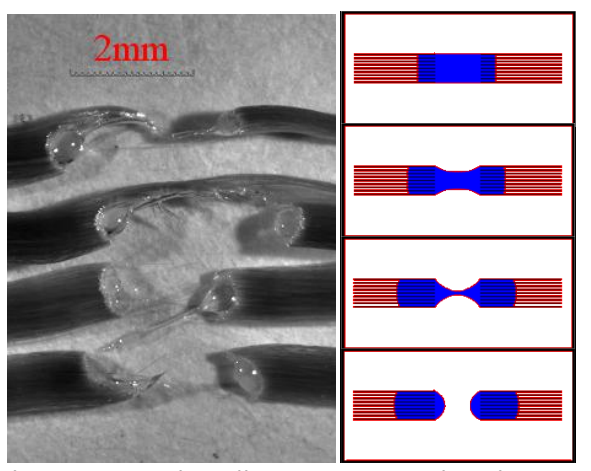

Figure 3 L: Characteristic bundle separation when laser irradiation is applied to a constrained bundle away from a free end. R: LBE output of fiber separation $(400 \times 800 \mu \mathrm{m})$.

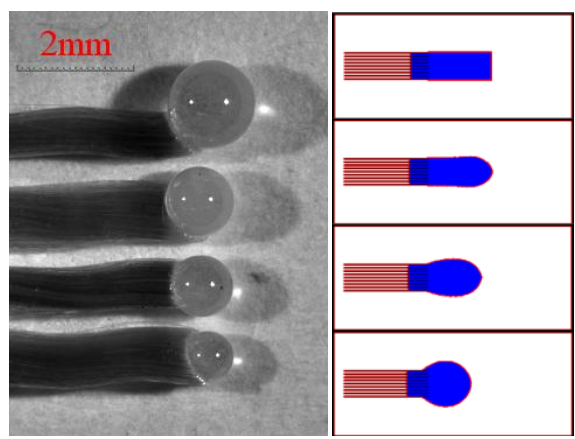

Figure $4 \mathrm{~L}$ : Characteristic bead formations when laser irradiation is applied to an unconstrained bundle near a free end. The bead volume is shown as a function of decreasing scanning distance from top to bottom. R: LBE model of a single fiber bundle irradiated near a free end $(400 \times 800 \mu \mathrm{m})$. 
Joining of individual fibers within a bundle into a spherical bead allows for the handling and subsequent joining of multiple bundles because the size of each bead is sufficient to bridge the gap between multiple bundles. Precise manipulation of laser power, focus and position is required to control the local temperature distribution of the sample, thereby controlling the densification and flow behavior of the fiber compact. Bead formation processes investigated here develop the fundamental understanding to overcome capillary and density induced flow effects for the joining of $3 \mathrm{~d}$ preforms. Joining of fiber bundles within a woven structure requires additional fiber volume in order to prevent void formation. Joining of $3 \mathrm{~d}$ woven pre-forms will require the formation of dense structures in the centers of fiber bundles, involving local irradiation and densification of the joint region prior to laser irradiation.

\section{Surface Tension and Capilarity Induced Flow}

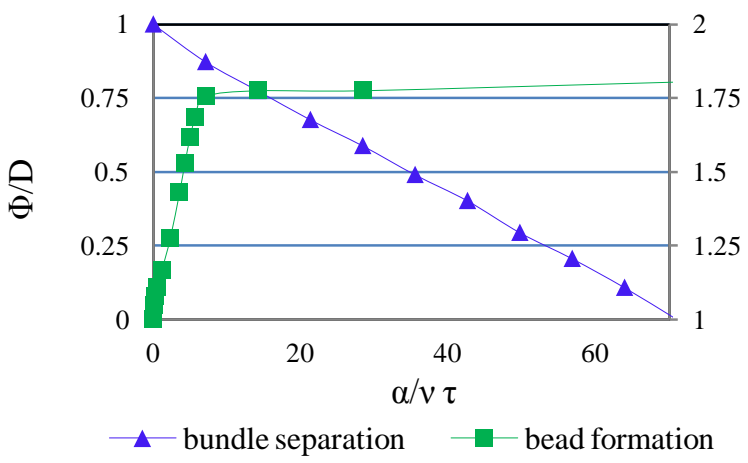

Figure 5: Normalized joint diameter versus surface tension and viscosity normalized time history from LBE model. Bundle separation references the left axis while bead formation references the right axis. All diameters are normalized by the initial pool diameter.

\section{Bundle Joining}

Fiber bundle joining was achieved by irradiating and connecting two bead structures from two pre-processed fiber bundles. The undesired flow of the individual bundles was overcome by bringing two processed beads into contact during the heating process. It was observed that because of the higher absorption of the bead structures, lower laser intensity $\left(10 \mathrm{~W} / \mathrm{mm}^{2}\right)$ was required to achieve the same melt consistency as the bead preparation step. Upon contact the flow pattern of each bead was dominated by both the capillary force at the fiber/melt interface and at the contact point between the beads.

Given sufficient initial volume, the two beads will rapidly coalesce, applying a tensile force on the two fiber bundles. If the bundles are unconstrained, the resulting joint will achieve a spherical profile, similar to the two initial beads. If the two bundles are constrained along their axis, the competing tensile forces acting on the melt pool will result in an ellipsoidal joint geometry. The resulting joint morphologies are depicted in Figure 6. The time history of this process is depicted in Figure 7. Note from the time history of the joining process that the fluid flow is initially dominated by the high surface area near the contact then by the bundle separation behavior observed in a single bundle. Similar to the initial bead process, the volume of the final joint is a function of the volume of fiber consumed in the joining process. Joint formation was found to occur at an average power output of 30 to $40 \mathrm{~W}$ laser exposure for one to two seconds.

Applying tension on joined fiber bundles while cooling results in dramatic joint elongation. Spherical, elliptical and cylindrical or conically tapered morphologies are observed at various tensile loads applied during cooling, as depicted in Figure 8. While the joint morphology is affected most readily along the axis of the joint, SEM images of the fracture surface (Figure 9) show that the fibers within the bundle also compacted in the radial direction when stretched. Fiber fusion joining of $3 \mathrm{~d}$ pre-forms is expected to take place in much the same manner as fiber fusion in single bundle joining. Although through thickness joints involve the fusion of adjacent bundles perpendicular to the fiber axis, the flow and compaction of the joints in a $3 \mathrm{~d}$ pre-form is expected to follow the same characteristic behavior as that observed in single bundle experiments.

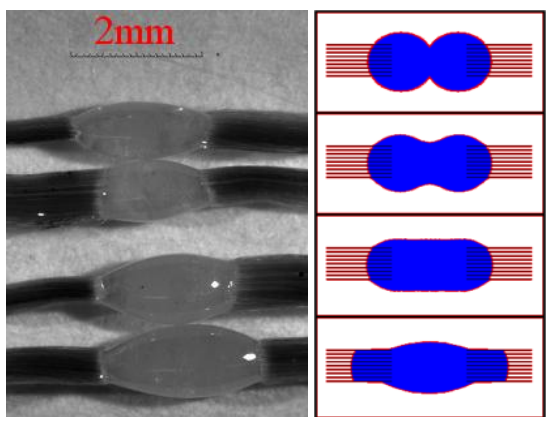

Figure 6: A: Single joints of constrained bundles obtained experimentally and from LBE simulations. B: LBE output of bead joint formation $(400 \times 800 \mu \mathrm{m})$.

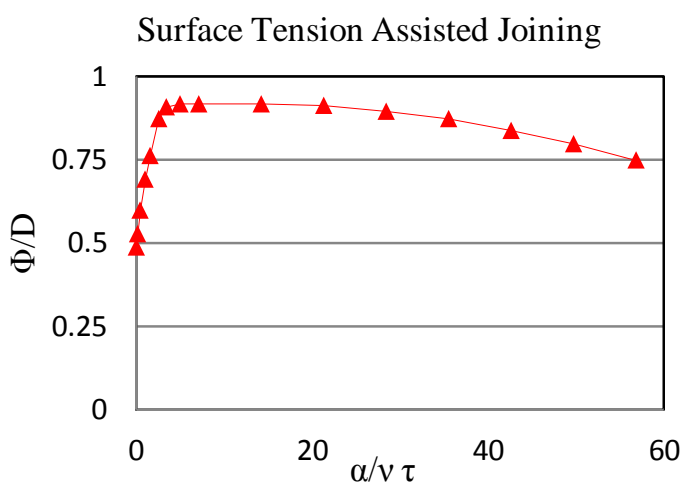

Figure 7: Normalized joint diameter versus surface tension and viscosity normalized time history from bundle joining LBE model. Note there is a fast time scale similar to bead formation and a slow time scale dominated by bundle separation.

\section{Bundle joint strength}

Laser joining of fiber bundles introduced microscopic voids in the bead, as observed through optical microscope images. It was observed from repeated tensile tests that joint failure consistently occurs near the joint rather than through 
the joint. Fibers at the periphery of the joint interface consistently fractured at the lowest loads. The failure mechanism of the fiber joint is found to be dependent upon the joint geometry and the fiber packing fraction near the joint rather than voids or micro cracks within the joint. No micro cracks were observed within the joint from either optical or scanning electron microscopy images. From these observations, it is expected that the joining process introduces a stress concentration in the fibers closest to the edge of the joints. Given the spherical or ellipsoidal morphology of the joints tested, the contact angle between the fiber and the joint is considered as the cause of the stress concentration.

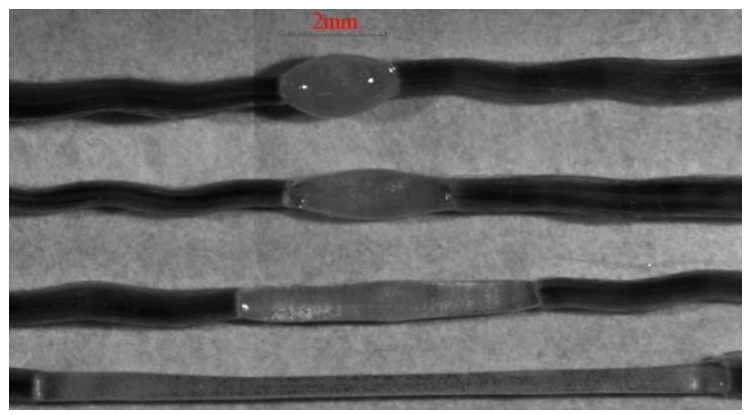

Figure 8: Joint morphologies obtained from various degrees of fiber constraint and tension. Joint length and fiber packing are both affected by bundle tension.

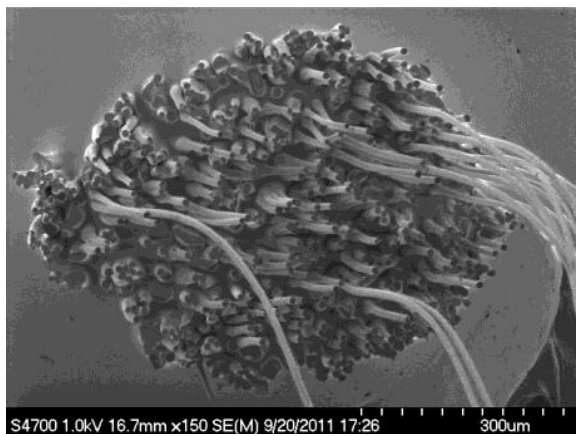

Figure 9A: SEM image of high density fiber packing at the joint interface. Fiber density is found to be controlled by the bundle confinement and tension during the joining process.

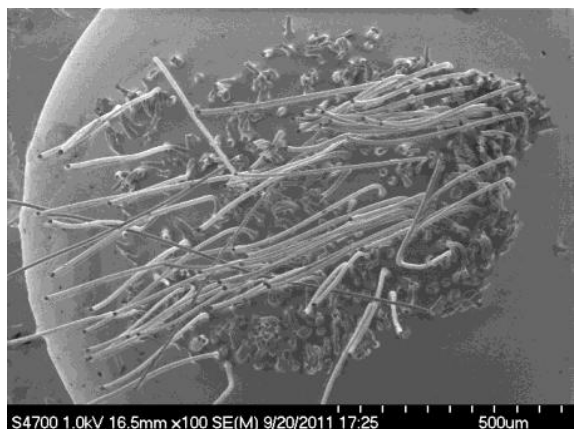

Figure 9B: SEM image of low density fiber packing at the joint interface. Low fiber packing density is found to be a source of added stress concentration resulting in lower loads at failure observed during tensile tests.

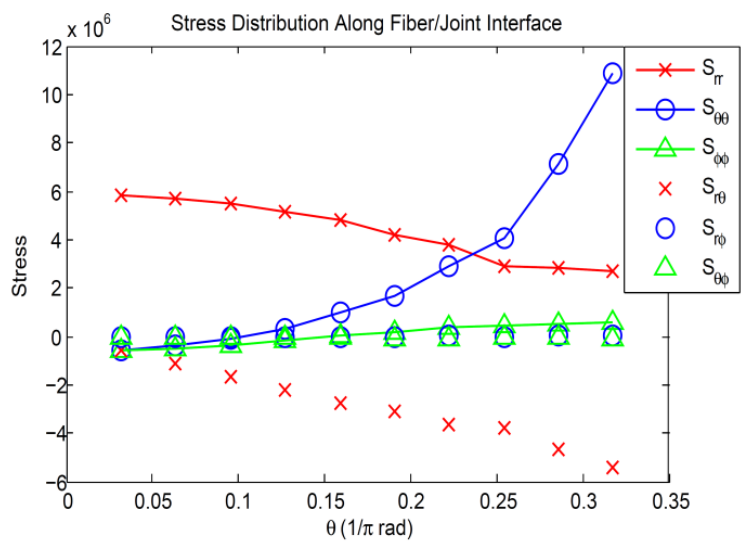

Figure 10: Stress state at the joint interface in spherical coordinates in angle $\boldsymbol{\theta}$ along the bundle radius. The stress concentration at the joint periphery is found to be a function of the contact angle $\boldsymbol{\theta}$.

Finite element modeling of the stress state in the joint showed a significant stress concentration at the periphery of the joint, as depicted in Figure 10. A weak relation is observed between the contact angle and stress concentration at a factor of two, in Figure 11. Tensile test results compared well with finite element findings, yielding a maximum load of $50 \%$ of the stock fiber material. Effects of bundle misalignment, joint defects, fiber packing and fiber defects are not accounted for in this finite element model. The presence of defects is apparent in the wide range of tensile test data. Variation in fiber packing fraction, as depicted in Figure 9, is investigated. Compiling tensile test results by fiber bundle diameters measured under cotical microscopy, an inverse trend is observed between packing diameter and critical load, as shown in Figure 12.

Examination of the fiber fracture surfaces under SEM have shown two distinct fracture mechanisms between the fibers near the center of the joint and those at the periphery of the joint. As observed in Figure 13A the fibers in the center of the joint exhibit a flat crack surface normal to the axis of the fiber, indicative of brittle fracture under uni-axial tensile loading. Fibers on the periphery of the joint, depicted in Figure 13B, exhibit a concave crack with a normal skewed away from the fiber axis. These findings agree with the shear stress concentration at the joint perimeter predicted in the finite element solution.

From numerical and experimental results, the sources of stress concentrations in the fibers and the fracture mechanism have been identified. A maximum strength of $50 \%$ is observed for the joint geometry achievable by the proposed method. The fracture mechanism of the joint is controlled by the stress concentration due to the contact angle and fiber compaction at the joint interface. It has been shown that all mechanical and adhesive composite joining methods impart some degree of stress concentration in the material [27,28]. Crack initiation and failure mechanisms at joint locations govern the macroscopic strength and failure of the composite $[28,29]$. As has been observed in this study, the joint morphology and fiber packing achieved during processing will determine the strength and failure characteristics of the composite. Reinforcement joining between adjacent layers 
within $3 \mathrm{~d}$ fiber pre-forms will be the dominant mechanism by which through thickness loads is transmitted. Contact angle and packing density at the joint interface will thus determine the out of plane strength and fracture behavior of the finished composite.

Contact Angle Induced Stress

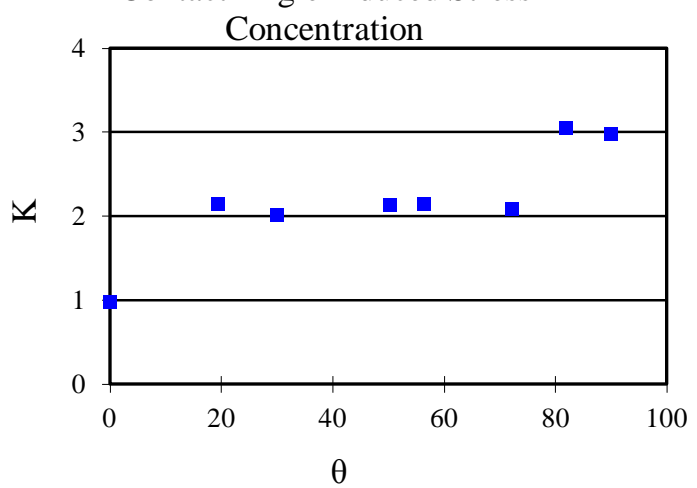

Figure 11: Stress concentration factor as a function of the maximum contact angle $\boldsymbol{\theta}$ obtained from finite element simulations of an idealized joint morphology. The factor of two stress concentration obtained numerically agrees with the strength reduction observed from uni-axial tensile tests of single bundle.

\section{Critical load vs Fiber Compaction}
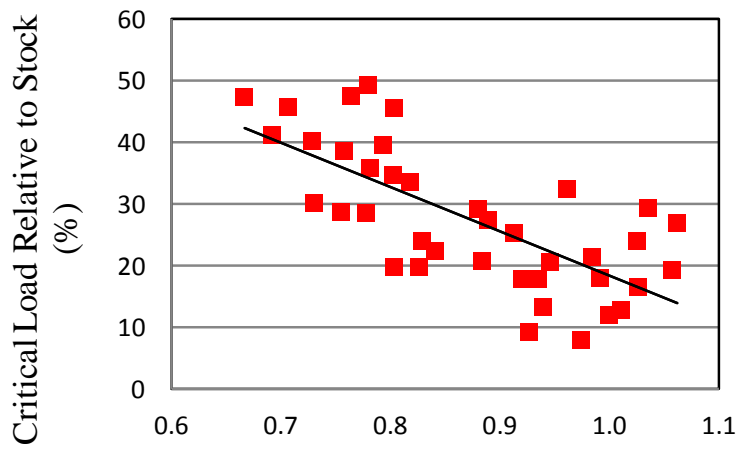

Bundle Diameter (mm)

Figure 12: Fiber compaction effect on strength experimentally obtained critical strength. Taking the fiber count within the bundle to be constant, fiber compaction is directly a function of bundle diameter observed under optical microscopy.

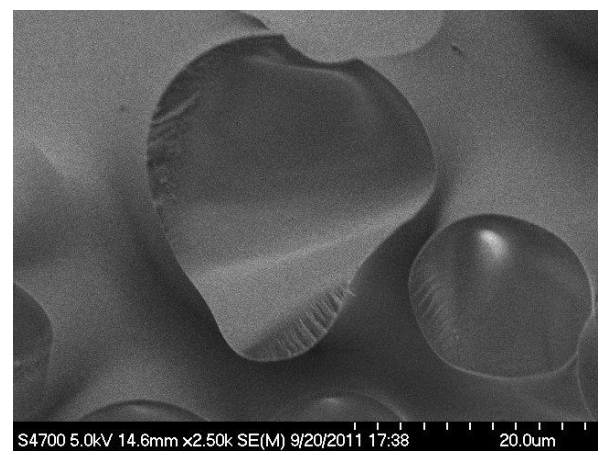

Figure 13A: Fiber fracture surfaces observed from the periphery of a joint. A concave, angled fracture surface here is indicative of a shear dominant failure mechanism at the stress concentration region.

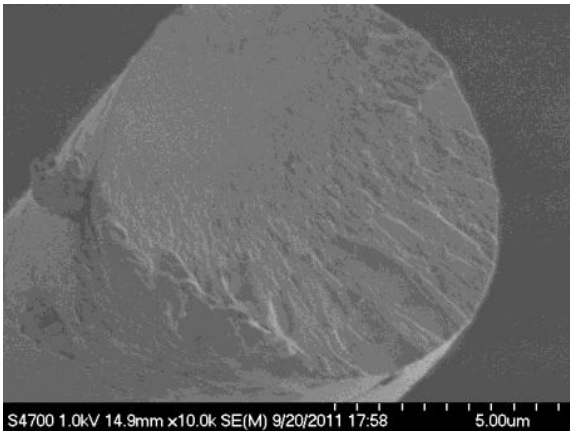

Figure13B: Fiber fracture surface found the center of a joint. A flat fracture surface is indicative of a brittle fracture due to axial tension.

\section{Seam joint strength}

To evaluate the application of the fiber joining process for more complex composite pre-forms, an extension of the process is used to join two plain weave fabric swatches. A seam joint between two linear arrays of opposing bundles is produced using the same two step beading and joining process described in the single joint method (Figure 14). Each swatch is prepared by first forming beads along the joining edge. The samples are then brought together such that the bundles were aligned axially along the joining edge. The laser spot is defocused and passed along the joint edge, fusing each pair of beads into a separate joint. During this process, the alignment and proximity of each bead was critical in determining the final joint geometry and strength.

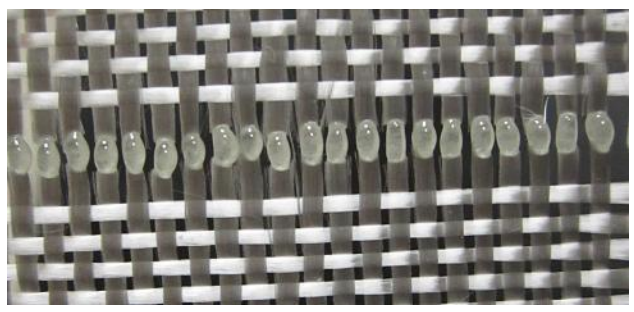

Figure 14: Seam joint between individual joints for each opposing fiber bundle. Crack initiation and propagation occurred much earlier in co-joined fiber bundles than in individually joined fiber bundles.

Tensile tests of seam welded samples showed the same failure initiation and propagation mechanisms as that observed in the single bundle tests. Raw fabric exhibited breaking loads of $75 \%$ that of a single bundle. The observed reduction in strength is due to the crimped nature of plain weave fabrics as has often been cited in composite mechanics literature [30]. During seam joint tensile tests, fiber compaction had a significant effect on the seam strength. Alignment and fixture inaccuracies during the welding process further complicated the loading geometry of the weld joint, further reducing the strength. It was observed that a single, extended joint along the length of the edge promoted the propagation of a single crack along the periphery of the entire joint. Samples exhibiting this mechanism yielded the lowest strengths 
observed during testing. The maximum strength of a seam weld is $30 \%$ of the stock fabric material, as shown in Figure 15 .

The purpose of demonstrating a seam joint between two plain weave glass fabrics is not to suggest that such a joint would be desirable or beneficial in a composite application. The seam joint is a simple extension of the single joint process developed and is selected for its direct relation to the single joint test data. Fiber fusion joining of $3 \mathrm{~d}$ fiber pre-forms should be considered in regions of existing stress concentration or material discontinuities. Material discontinuities commonly result either by design or from manufacturing processes. Examples of possible joining candidates include composite free edges [27], internal ply drop offs [29,31], holes [32] and between layers of lamina[33]. The potential for joining damaged fibers may also be of interest in the repair of composite structures after low velocity impacts [34].

Fiber to fiber mechanical connections derived from laser fusion joining method developed in this work has been shown to yield strengths on the order of the original glass fiber. Through thickness joined $3 \mathrm{~d}$ pre-forms composed of repeated joints consisting of radial fused fiber bundles may yield far better strength and fracture characteristics than mechanical reinforcement methods such as pinning, stitching or weaving. Radial fiber joining may be achieved in the same two step process outlined in this work. Strength and failure characteristics of fusion joined fiber reinforcement pre-forms are expected to be strongly dependent on the contact angle and fiber packing fraction at the joint interface. In order to provide high through thickness strength, further investigation into laser fusion processing should focus on fiber packing and fiber contact angles between radially joined fiber bundles.

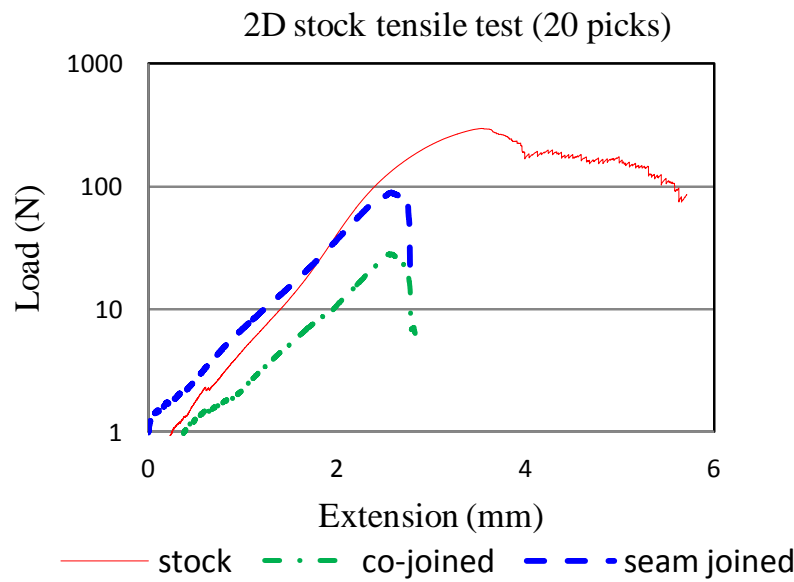

Figure 15: Tensile results of continuous plain weave fiber pre-form with and without seam joints. Joint samples of separate joint morphologies have been shown to achieve $30 \%$ of the critical load obtained by the stock woven material.

\section{CONCLUSIONS}

Laser fusion joining of glass fiber reinforcements has been achieved with a two step process, overcoming several obstacles to fiber joining inherent in the compaction, flow and relative density of glass fiber pre-forms. In order to bridge the gap between two fiber bundles, a bead formation and joining process is necessary to overcome the tendency of the material to flow away from the heat source. The magnitude and time dependence of the fusion process is governed by surface tension and viscosity: both of which are dependent upon temperature. The direction of capillary induced flow is governed by the initial pore geometry and relative density of the fiber compact. These findings, along with the numerical models implemented, provide the processing knowledge necessary to produce fiber to fiber joints between adjacent fiber bundles and lamina, leading to $3 \mathrm{~d}$ fiber pre-form joining.

Joint testing and finite element analysis reveal the internal stress state of joined bundles, yielding information on the effect of morphology on the stress concentration factor and effective strength of the joint. It is shown that the joint strength is highly dependent on the joint morphology and fiber packing. Stress concentration factors due to contact angle and bundle density are expected to play a major role in determining the strength and out of plane characteristics of laser joined $3 \mathrm{~d}$ fiber pre-forms.

Modeling methods for the heat transfer and sintering of fiber compact systems have been discussed and a Lattice Boltzmann bulk flow simulation has been implemented, showing good agreement with experimental observations. Further development of these models will help to better understand the physical limitations of the fiber joining process and guide future efforts in the joining of $3 \mathrm{~d}$ fiber pre-form architectures.

A fundamental investigation of the fiber fusion mechanism has been performed to evaluate the capability of laser fusion joining processing for the fabrication of $3 \mathrm{~d}$ fiber pre-forms. Fusion joined fibers exhibit tensile strengths of the same order as stock fibers, leading to potential strength gains in the replacement of mechanical fasteners in the assembly of $3 \mathrm{~d}$ composite pre-forms. Radial joint formation will involve its own material and manufacturing challenges, but will be based on the same processing principles and practices as those introduced in the axial joining of fibers.

\section{ACKNOWLEDGMENT}

Partial financial support from Columbia University is gratefully acknowledged.

\section{REFERENCES}

[1] Byrd L., and Birman V., 2006, "Effectiveness of z-pins in preventing delamination of co-cured composite joints on the example of a double cantilever test," Composites Part B: Engineering, 37(4-5), pp. 365-378.

[2] Huang H., and Waas A. M., 2009, “Compressive response of Z-pinned woven glass fiber textile composite laminates: Experiments," Composites Science and Technology, 69(14), pp. 2331-2337.

[3] Bilisik K., 2009, "Multiaxis 3d woven preform and properties of multaxis $3 \mathrm{~d}$ woven and $3 \mathrm{~d}$ orthogonal woven carbon/epoxy composites," Journal of Reinforced Plastics and Composites, 29(8), pp. 1173-1186. 
[4] Brandt J., 1996, "Mechanical performance of composites based on various three-dimensional woven-fibre preforms," Composites Science and Technology, 56(3), pp. 381-386.

[5] Bogdanovich A. E., 2006, “Advancements in manufacturing and applications of 3-d woven preforms and composites," 16th international conference on composite materials, pp. 1-10.

[6] Mohamed M. H., and Wetzel K. K., 2006, "3D woven carbon/glass hybrid spar cap for wind turbine rotor blade," Journal of Solar Energy Engineering, 128(4), p. 562.

[7] Cox B. N., and Dadkhah M. S., 1995, "The macroscopic elasticity of $3 \mathrm{~d}$ woven composites," Journal of Composite Materials, 29(6), pp. 785-819.

[8] Jones I., 2005, "Improving productivity and quality with laser seaming of fabrics," Technical Textiles International, (May), pp. 35-38.

[9] Niebel V., Weinert N., Gries T., and Seliger G., 2009, "Technology for overlap-free joining of semi-finished textile products," Technical Textiles, 52(2), pp. E68-69.

[10] Vatterodt T., 2006, "Sewing without thread - textile welding," Kunststoff, Plast Europe, 96(10), pp. 221-4.

[11] Scherer G. W., 1979, "Sintering of inhomogenous glasses: application to optical waveguides," Journal of Non-Crystalline Solids, 34, pp. 239-256.

[12] Rahaman M. N., Jonghe L. C. De, Scherer G. W., and Brook R. J., 1987, "Creep and Densification During Sintering of Glass Powder Compacts," Journal of the American Ceramic Society, 70(10), pp. 766-774.

[13] Chen J. C., and Churchill S. W., 1963, "Radiant heat transfer in packed beds," AIChE Journal, 9(1), pp. 35-41.

[14] Sun W., Loeb N. G., and Fu Q., 2002, "Finite-difference time-domain solution of light scattering and absorption by particles in an absorbing medium," Applied Optics, 41(27), pp. 5728-5743.

[15] Wedding B., 1974, "Measurements of high-temperature absorption coefficients of glasses," Journal of the American Ceramic Society, 58(3-4), pp. 102-105.

[16] Kask N. E., Radchenko V. V., Fedorov G. M., and Chopornyak D. B., 1979, "Temperature dependence of the absorption coefficient of optical glasses exposed to laser radiation," Nuclear Physics, 9(2), pp. 193-198.

[17] Gardon R., 1954, "Review of radiant heat transfer in glass," Journal of the American Ceramic Society, 44(7), pp. 305-312.

[18] Dvurechensky A. V., Petrov V. A., and Reznik V. Y., 1979, "Spectral emissivity and absorption coefficient of silica glass at extremely high temperatures in the semitransparent region," Infrared Physics, 19, pp. 465469.

[19] Kuczynski G. C., 1949, "Study of the sintering of glass," Journal of Applied Physics, 20(12), pp. 1160-1163.
[20] Kingery W. D., and Berg M., 1951, "Study of the Initial Stages of Sintering Solids by Viscous Flow, EvaporationCondensation, and Self-Diffusion," Journal of Applied Physics, 3, pp. 1205-1212.

[21] Succi S., 2001, The Lattice Boltzmann equation for fluid dynamics and beyond, Oxford Science Publications, Oxford.

[22] Rothman D. H., and Keller J. M., 1988, "Immiscible cellular-automaton fluids," Journal of Statistical Physics, 52(3-4), pp. 1119-1127.

[23] Lishchuk S., Care C., and Halliday I., 2003, "Lattice Boltzmann algorithm for surface tension with greatly reduced microcurrents," Physical Review E, 67(3), pp. 15.

[24] Gunstensen A. K., and Rothman D. H., 1991, "Lattice Boltzmann model of immiscible fluids," Physical Review A, 43(8), pp. 4320-4327.

[25] Scherer G. W., 1991, "cell models for viscous sintering," Journal of the American Ceramic Society, 74(7), pp. 1523-1531.

[26] Evans A. G., 1982, "Considerations of inhomogeneity effects in sintering," Journal of the American Ceramic Society, 65(10), pp. 497-501.

[27] Mittelstedt C., and Becker W., 2004, "Interlaminar stress concentrations in layered structures: Part I - a selective literature survey on the free-edge effect since 1967," Journal of Composite Materials, 38(12), pp. 1037-1062.

[28] Glaessgen E. H., Raju I. S., Poe C. C., Dynamics S., and Conference M., 1999, "delamination and stitched flailure in stitched composite joints," (99).

[29] Shim D. J., 1997, "Role of delamination and interlaminar fatigue in the failure of laminates with ply dropoffs," Massachusetts Institute of Technology.

[30] Bogdanovich A. E., Dannemann M., Döll J., Leschik T., Singletary J. N., and Hufenbach W. a, 2011, "Experimental study of joining thick composites reinforced with non-crimp 3D orthogonal woven E-glass fabrics," Composites Part A: Applied Science and Manufacturing, 42(8), pp. 896-905.

[31] Her S., 2002, "Stress analysis of ply drop-off in composite structures," Composite Structures, 57, pp. 235244.

[32] Young T., and Odriscoll D., 2002, "Impact of Nd-YAG laser drilled holes on the strength and stiffness of laminar flow carbon fibre reinforced composite panels," Composites Part A: Applied Science and Manufacturing, 33(1), pp. 1-9.

[33] Sela N., and Ishai O., 1989, "Interlaminar fracture toughness and toughening of laminated composite materials: a review," Composites, 20(5), pp. 423-435.

[34] Fischer F., Romoli L., and Kling R., 2010, "Laser-based repair of carbon fiber reinforced plastics," CIRP Annals Manufacturing Technology, 59(1), pp. 203-206. 